
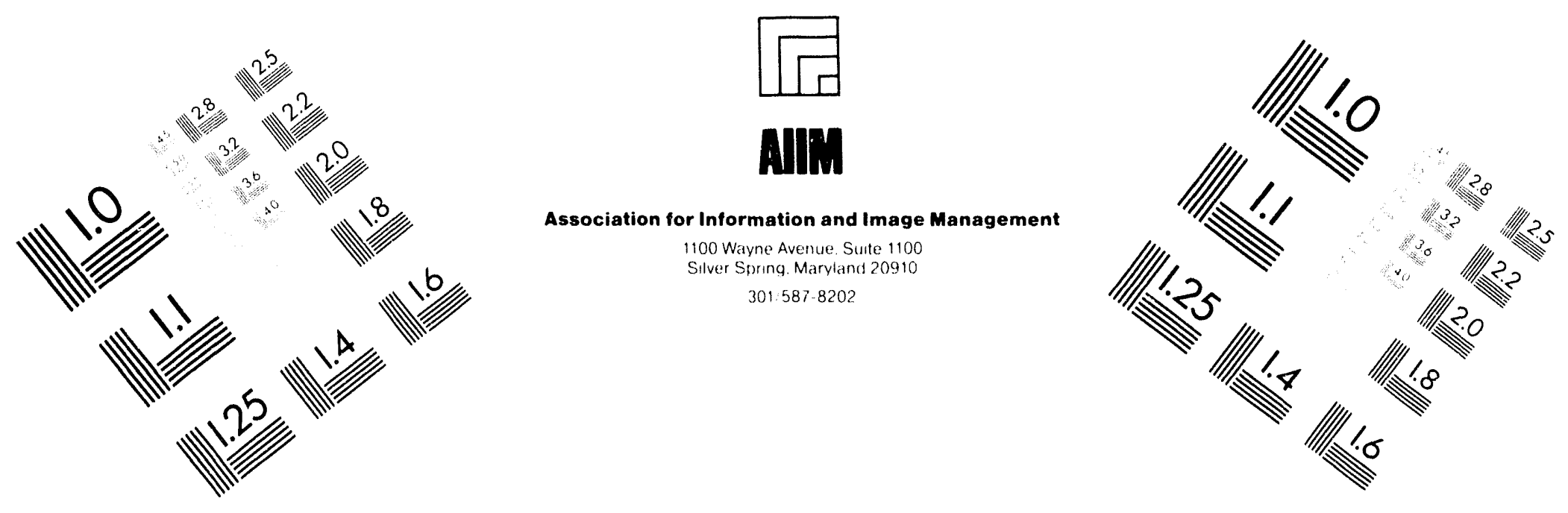

\title{
Centimeter
}

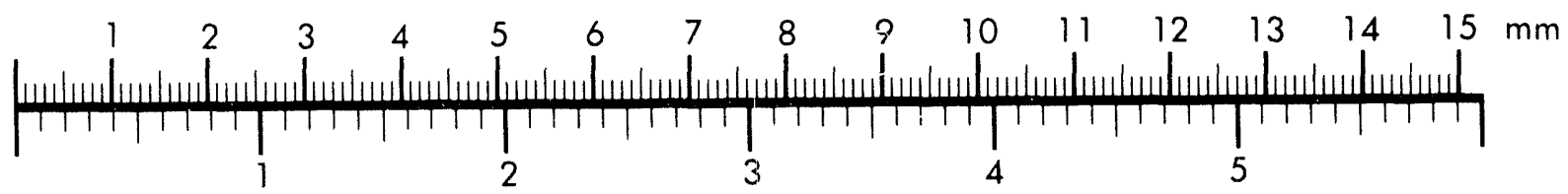

Inches
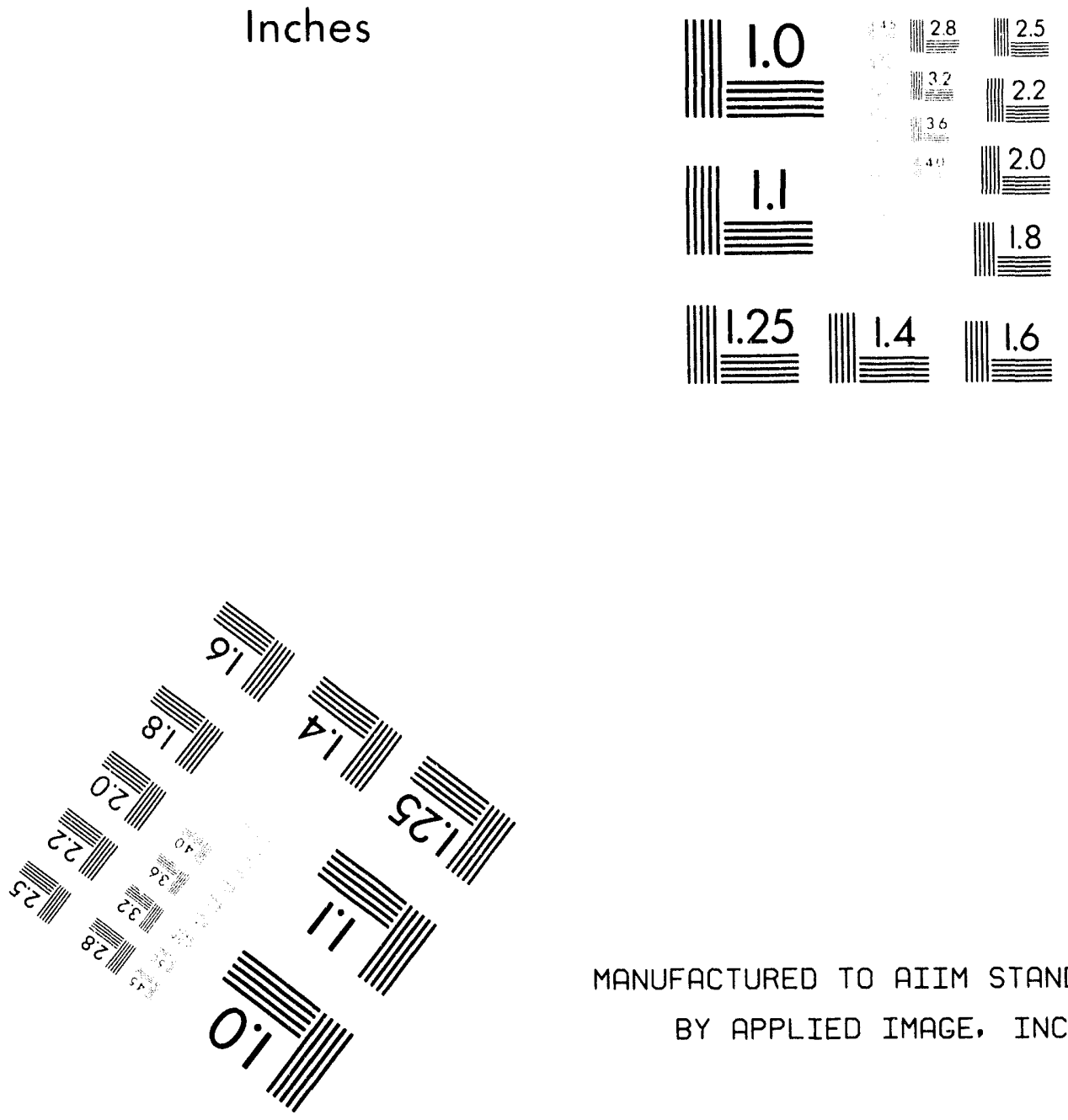

MANUFACTURED TO AIIM STANDARDS

BY APPLIED IMAGE. INC.

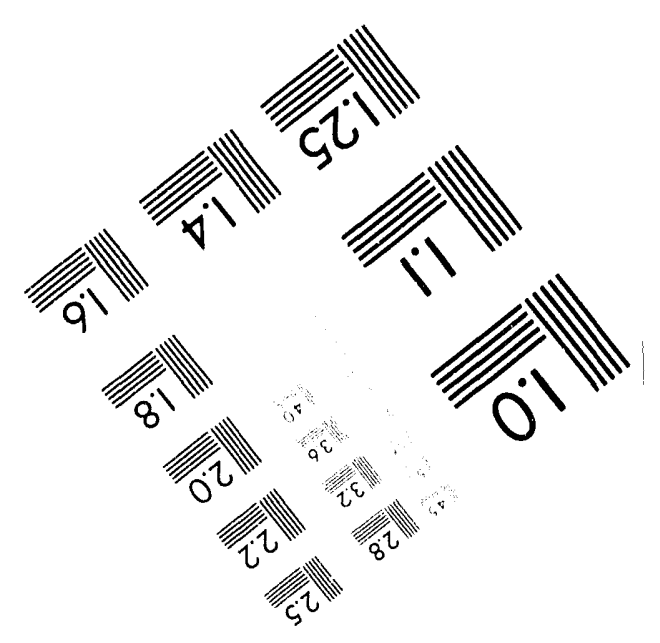



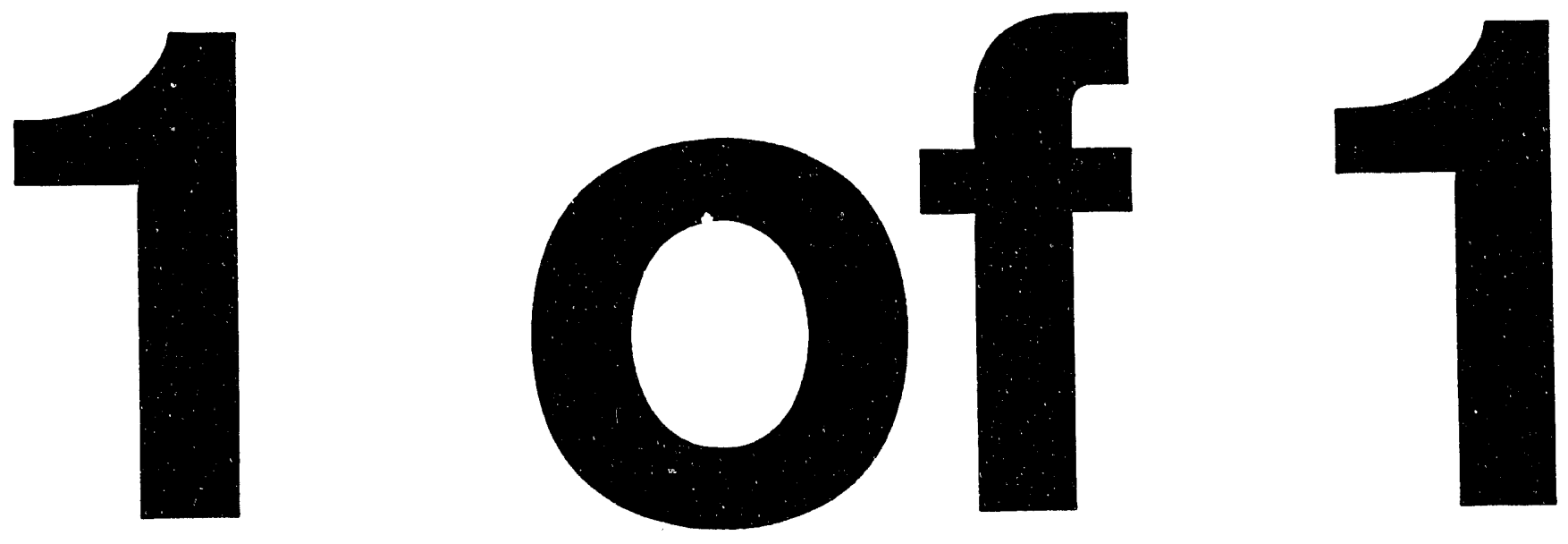


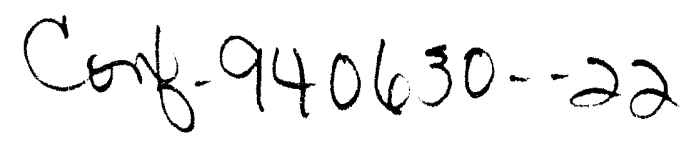

LA-UR- 94-1887

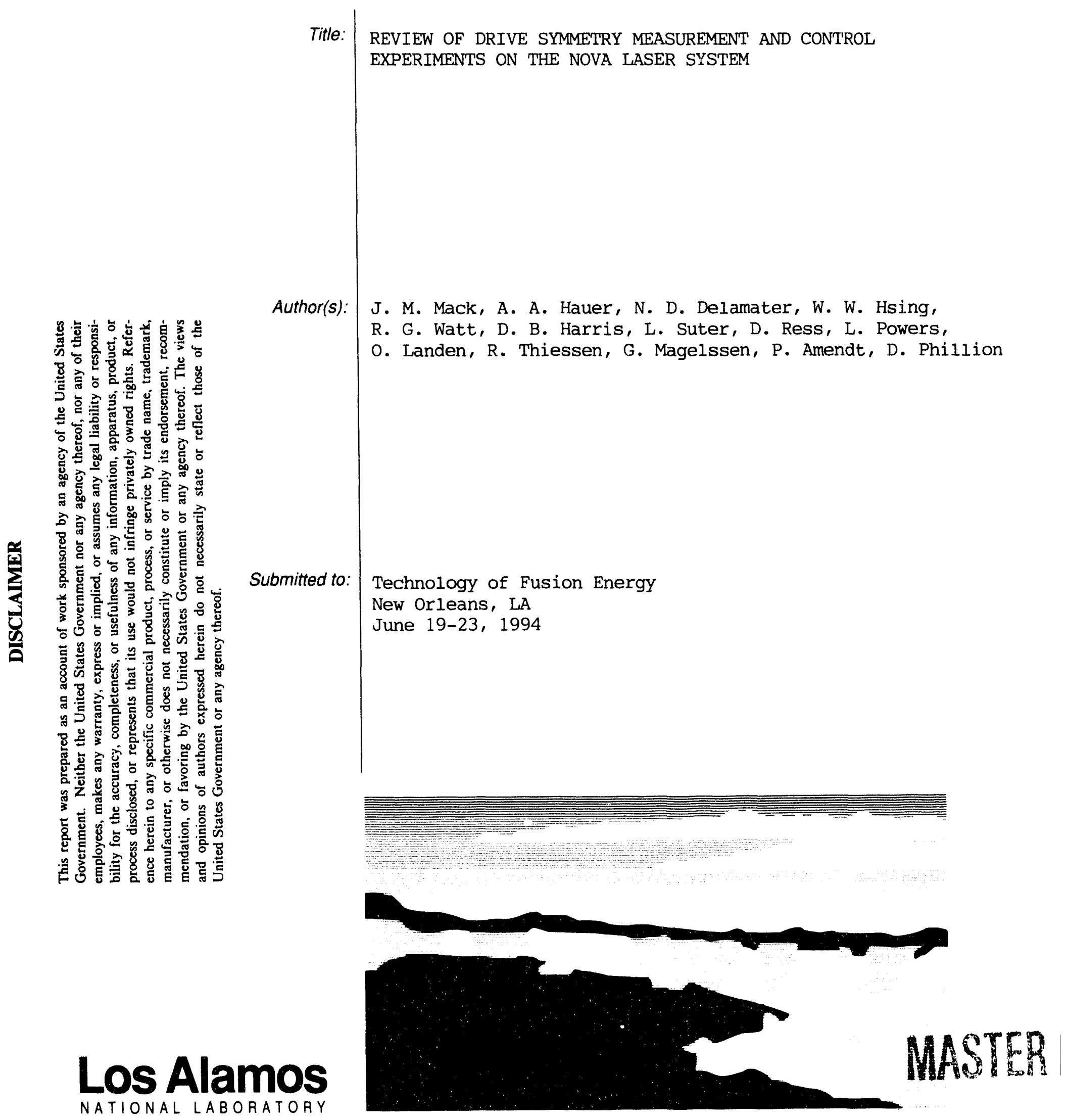

Los Alamos National Laboratory, an aftirmative action/equal opportunity emplóyer, is operated by the University of California for the U.S. Department of Energy under contract W-7405-ENG-36. By acceptance of this article, the publisher recognizes that the U.S. Government retains a nonexclusive, royalty-free license to publish or reproduce the published form of this contribution, or to allow others to do so, for U.S. Government purposes. Tne Los Alamos National Laboratory requests that the publisher identity this anticle as work performed under the auspices of the U.S. Department of Energy. 


\section{REVIEW OF DRIVE SYMMETRY MEASUREMENT AND CONTROL EXPERIMENTS ON THE NOVA LASER SYSTEM}

\author{
J. M. Mack, A. A. Hauer, N. D. Delamater, \\ W. W. Hsing, R. G. Watt, D. B. Harris \\ Los Alamos National Laboratory \\ P. O. Box 1663 \\ Los Alamos, NM 87545 \\ (505) 667-3416
}

\author{
L. Suter, D. Ress, L. Powers, O. Landen, \\ R. Thiessen, D. Phillion, P. Amendt, \\ G. Magelssen \\ Lawrence Livermore National Laboratory \\ P. O. Box 808 \\ Livermore, CA 94551
}

\begin{abstract}
ABSTRAC'T
Symmetric radiation drive is required for achieving ignition in laboratory experiments. Over the last two years, concerted series of drive symmetry experiments have been performed on the Nova laser system. The goals of this work were to develop measurement techniques and to apply them to symmetry variation and control experiments.
\end{abstract}

The emphasis in this initial work has been on time integrated measurements (integrated over the laser drive pulse). We have also begun work on methods for time resolved measurements. Most of our work used the symmetry signature impressed on the compressed core of a capsule imploded in a hohlraum (cylindrical cannister) environment. X-ray imaging of this core provides a mapping that can be compared with theoretical modeling and related to a specific amount of drive asymmetry. This method is indirect and we have taken great care in understanding the formation of the symmetry signature and in its comparison with simulations. A review of drive symmetry measurement and control experiments is presented, including data from time integrated and time resolved measurements; these measurements are also compared to modeling. Under carefully controlled conditions results from symmetry measurements (and from other auxiliary measurements) are reproducible, and indicate that aspects of implosions symmetry can be controlled.

\section{INTRODUCTION}

Indirect drive laser fusion occurs in an enclosure known as a hohlraum (usually made out of a high $\mathrm{Z}$ material such as gold) that is heated internally by the absorption of laser energy on its inner wall. A typical illumination geometry for Nova hohlruam experiments is shown in Figure 1.
The five beams coming from each side form 2 cones that can be thought of as illuminating the hohlraum with 2 "rings" of light. The regions that are directly illuminated by the laser are initially the dominant source of soft $x$-ray radiation. X-rays from these spots illuminate the rest of interior, the walls heat up, and the $x$-ray sources become more evenly distributed. However, the regions irradiated by the laser are always somewhat hotter than the surrounding walls. As wall material blows off and begins to fill the hohlraum, regions of laser absorption can move radially inward. This motion is generally referred to as "spot motion". The polar symmetry (the polar axis being the cylindrical axis of the hohlraum) is controlled by moving the beam "rings" with respect to the plane of the capsule. Also, shown in Figure 1 are typical dimensions used in the present symmetry studies.

Time integrated symmetry (integrated over the entire length of the drive) was of initial primary concern for ignition level target performance. However, we have also been developing methods for measuring symmetry as a function of the laser-pulse time history. The following techniques for symmetry measurement have been studied:

$X$-ray imaging of the self emission from the stagnating core of imploded targets, which provides a signature of the drive symmetry imposed on the capsule; ${ }^{1}$

X-ray imaging of the "sources" inside a hohlraum though soft $x$-ray "spot motion" studies; ${ }^{2}$ hard $x$-ray imaging using thin walled hohlraums; 3 
direct imaging of re-emission from a surrogate target; ${ }^{4}$ and

backlighting of the shocks driven into a surrogate "witness ball" target. ${ }^{2}$

\section{TIME INTEGRATED METHODS}

In the most mature method of assessing the radiation field symmetry in the hohlraum, a capsule is placed in the center and is imploded by $x$-ray drive. X-ray imaging is performed on the compressed core and the shape of the image reflects the time integrated drive asymmetry experienced by the capsule. The radiation field impresses a characteristic symmetry signature on the compressed core. The basic measurement configuration is illustrated in Figure 2. X-ray imaging is performed in both time integrated and time resolved modes along two or more lines of sight. Time resolved imaging is done with gated micro channel plate detectors which give a series of frames of about 80 ps. duration. This provides an emission history from the stagnated compressed core. The implosions used in this work have a low convergence ratio $(\sim 8-10)$, so that hydrodynamic break up of the shell does not affect the data.

Time integrated images can also be accurately calibrated to within several different spectral channels. This diagnostic package provides a more detailed picture of the core emission, very useful in comparing with theoretical modeling. The image data is processed to provide intensity contours that can be used to infer the flux asymmetry by comparisons with integrated hydrodynamic modeling of the hohlraum environment and the capsule implosion. In Figure 3 we illustrate the process used in inferring the flux asymmetry from the 2D image data. With respect to the data shown in Figure 3, it should be noted that a $2: 1$ distortion is caused by less than a $7 \%$ flux asymmetry. Distortions much less than $2: 1$ (eg. $\sim 1: 1$ ) can be sensed, which (from the modeling in Figure 3 ) implies that asymmetries less than $2 \%$ can be detected. In addition to X-ray imaging, symmetry diagnosis was also measured by other methods. As shown in Figure 2, the gas fill in the center of the target contains both DD and argon gas. Nuclear yield (and its time history) and $\mathrm{x}$-ray spectra from the argon are also recorded, followed by detailed comparison with modeling. In this "feedback" process, as illustrated in Figure 2, we can critique and constrain the modeling, thereby providing added confidence in the symmetry determinations.
More detailed information can also be obtained from the image signature by decomposing the contours into spherical harmonic components (or other appropriate constructs). For example a negative $\mathrm{P}_{2}$ coefficient corresponds to an "elliptic-like" departure from a sphere and produces a compressed core that is flattened along the hohlraum axis. An example of this decomposition is given in Figure 4. The contour chosen corresponded to $50 \%$ of the peak exposure value.

The image in this case was shown to have significant $\mathrm{P}_{4}$ content, which results in a slight "squaring off" of the core. Hohlraums are effective in smoothing high frequency non-uniformity, but tuning is required to reduce the lower order modes. Correction of $\mathrm{P}_{2}, \mathrm{P}_{4}$, and $\mathrm{P}_{6}$ to less than $2 \%$, (time averaged) is required for ignition and the methods for accomplishing this are part of the National Ignition Facility (NIF) design.

These measurement techniques have been applied to a varicty of symmetry tuning and control experiments. The polar symmetry is changed by moving the beam cones relative to the capsule and characteristic changes in the image configuration are observed. A generic picture of the qualitative variation in capsule images is shown in Figure 5. Hotter sources of radiation are produced in the regions of laser deposition. At pointing locations nearer the capsule, these sources cause equator hot drive, producing what is referred to as a "sausaged" image. For pointing locations further from the capsule, the drive is pole hot, producing a "pancaked" image. For reference we also show the zero of the $\mathrm{P}_{2}$ mode. In plots of symmetry variation the position of the crossover point of the beams with respect to the capsule position (parameter d in Figure 1 ) is used as the reference.

We have studied this variation in symmetry under a variety of conditions:

I ns square and shaped (2.2 ns) laser pulses; and

pure gold hohlraums and gold lined with

$\sim 1700 \AA \mathrm{Ni}$ and other materials.

At extreme pointing conditions the length of the hohlraum was changed in order to avoid perturbation of the symmetry measurements by laser entrance hole (LEH) effects.

An example of the case studies performed with symmetry tuning is given in Figure 6. These data show only the " $\mathrm{P}_{2}$ - like" image distortion ratio $a / b$. These measurements were taken using a pure 
gold hohlraum driven by a shaped laser pulse approximately $2.2 \mathrm{~ns}$ in duration (the laser profile is shown in the Figure $6 \mathrm{~b}$. In Figure $6 \mathrm{a}$ the variation in image distortion, as measured by the simple parameter $\mathrm{a} / \mathrm{b}$, is plotted vs. heam cross-over position with respect to the capsule and compared with theoretical modeling. The modeling is based on an integrated simulation of the laser hohlraum interaction and the resulting $x$-ray driven implosion of the capsule employing the radiation hydrodynamic code Lasnex ${ }^{7}$. The simulation also includes a calculation of the $x$-ray image, which is compared in detail with the experimental data.

Errors in the measurement of image distortion (and thus the implied flux asymmetry) are indicated in Table 1. The "tilt" error arises when observing compressed cores that are distorted such as the "pancaked" (p) image in Figure 4. A distorted compressed core such as this can be "tilted" (or have more general 3 dimensionality) due to modest laser beam energy imbalances and beam pointing and timing in accuracies. Thuse can introduce an error in the determination of the polar symmetry. For the imaging views used in the present work, tilt of a $(p)$ image is more sensitive. For images in Figure 2 the left hand view can sense tilts in an (s) image, but observation of tilt in a (p) image would require a top (plan) view, which was not available.

\begin{tabular}{|l|l|}
\hline & $\begin{array}{l}\text { magnitude } \\
(\text { error in a/b) }\end{array}$ \\
\hline $\begin{array}{l}\text { "tilt of the image } \\
\text { or target }\end{array}$ & $\begin{array}{l}-2.5 \%(\mathrm{p}) /+1.5 \\
\%(\mathrm{~s})\end{array}$ \\
\hline image resolution & $\pm 2.5 \%$ \\
\hline beam pointing & $\begin{array}{l} \pm 4 \%(30 \\
\text { microns rms } \\
\text { error in pointing })\end{array}$ \\
\hline
\end{tabular}

Table 1 Approximate error budget for the determination of symmetry from image distortion (e.g.. a/b quantity). Image "tilt "refers to small perturbations in the compressed core (due e.g.. to beam imbalance) that cause departures from the $2 \mathrm{D}$ picture that is symmetric with the hohlraum axis. This tends to affect the "pancaked" image more strongly than the "sausaged". The values in the table are approximate and apply to modest distortions (i.c.. $\mathrm{a} / \mathrm{b} \sim 1)$ and are somewhat larger for larger image distortion.

A rotation $\theta$ of a (p) image around an axis perpendicular to the view of Figure 2 results in a reduction of the apparent ratio $a / b$ to: $\mathrm{a} /(\mathrm{a} \sin \theta+\mathrm{b} \cos \theta) \sim \mathrm{a} /(\mathrm{a} \theta+\mathrm{b})$. The fractional change in distortion is then $\sim(\theta)(a / b)$ measured. The image resolution is determined by the size of the imaging pinhole, the detector noise due to film and microchannel plates.

Beam pointing precision was established in a series of dedicated laser shots in thin-walled cylindrical hohlraums. The beams positions were determined by X-ray imaging of the laser spots on thin-walled, semi-transparant hohlraum walls. Pointing precision with a standard deviation of 30 $\mu \mathrm{m}$ was achieved in the hohlraums, using precision Nova aiming procedures with much care taken with regard to target positioning. Detailed results are reported in separate publications.

\section{TIME RESOLVED METHODS}

It is also important to measure the temporal fluctuation of the drive symmetry. As an example it will be necessary in ignition target experiments to sense fluctuations occurring on a I ns time scale and 10 percent amplitude. Such fluctuations could in principle average to zero over the entire drive interval, thus giving an approximately round implosion image. We now describe methods for sensing such fluctuations.

Implosion imaging is also used for time resolved studies when combined with methods for sampling a portion of the drive. "Symmetry capsules", one method ${ }^{6}$ of drive sampling, is illustrated in Figure 7. The capsules placed in the hohlraum environment have different initial wall thicknesses and therefore different implosion velocities. The rapidly imploding capsules sample only the early portions of the drive, as schematically illustrated in Figure 7.

Figure 8 illustrates the use of this technique in sampling the evolution of a hohlraum and capsule driven by a shaped laser pulse. In the early phase of a typical evolution the hohlraum wall regions outside the laser spots are at a significantly lower temperature than the regions directly heated by the laser. The hot spots under the laser beams dominate the X-ray drive causing an "equator-hot" condition inside the hohlraum. Data in Figure 8 b indicate early drive-interval samples through the use of a relatively fast imploding capsules. The image distorted along the hohlraum axis (i.e., "sausaged") is indicative of equator-hot drive. Figure 8a shows drive sampling using a capsule that is imploding more slowly. This implosion samples a longer interval over which the time-averaged symmetry is improved, resulting in a 
rounder image. At this interval the walls have heated more uniformly and some spot motion has occurred. Hydrodynamic simulation of the of the images in Figure 8 give a/b ratios of .96 and .68 , respectively.

Another method for measuring time-varying symmetry uses reemission from a non-imploding surrogate witness ball placed in the hohlraum at the nominal capsule location, as shown in Figure 9. A solid sphere is suspended in the hohlraum on a very tenuous plastic film $(\sim 1000 \AA)$ made of formvar. This sphere is heated by the hohlraum environment and reemits its own intrinsic radiation. Imaging this reemission can yicld a flux mapping at the ball position. Choosing the energy band in which to image is quite important. At low energies there is a competition with intrinsic emission from the hohlraum which acts as a noise background. Al higher energies the emission falls off exponentially and becomes difficult to detect.

To test this technique, an intentionally large laser beam east to west imbalance was purposefully formed, as is shown in Figure 9. This created a large initial radiation imbalance in the hohlraum, clearly reflected in the image of the emission from the surrogate sphere. In Figure 10 data from the reemission technique are used to give an example of the temporal evolution of the hohlraum environment. As in the case shown in Figure 9, intentional initial laser beam imbalance is observed, obvious in the early image frame. Later frames show the effect of the hohlraum's radiation redistribution that evolved into more uniform emission from the witness ball The redistribution is never perfect (because of such a large initial energy imbalance), and a residual imbalance remained. The hohlruam smoothes out imbalance to some degree, but there is still a requirement for establishing laser beam balance quality, needed to achieve desired drive conditions for high performance implosions.

The reemission technique is most useful in the early stages of the hohlraum evolution and has been shown to sense fluctuations similar to what will be required for ignition targets. For example, the evolution shown in Figure 10 illustrates that flux imbalances of a few percent can be detected over an interval of a few hundred picoseconds. Eventually, plasma filling within the hohlraum interacts with the ball and causes more complex emission patterns.

Another promising method for time resolved symmetry measurement is being developed ${ }^{5}$. This uses backlighting radiography of the shock driven into a low density foam target. Asymmetries in the drive result in distortions of the shock front which are measured and can be compared with modeling. Details of this method will be described elsewhere.

\section{GENERAL COMMENTS ON SYMMETRY CONDITIONS}

It is interesting to compare the strengths the implosion and reemission methods. The implosion method has the distinct advantage of what could be referred to as an amplification factor. The convergent geometry of the implosion converts differences in velocity (caused by different $x$-ray ablation rates) to differences in final displacement multiplied by the convergence ratio. Reemission has no such amplification and its observable is directly related to the flux pattern. Conversely, it is difficult with the implosion methods to study very early time hehavior, since there must be enough integrated flux to produce a strong, well characterized implosion. Therefore, reemission is well suited to early time measurements. Being directly sensitive to the flux it is not a robust implosion-drive sampling method, but provides a real time measurement of flux variation.

Comparison of experimental and modeling data demonstrates sensitive symmetry tuning that agrees with modeling is possible. For example variations in pointing of $\sim \pm 1 \%$ around the optimum pointing condition (round image) can be detected. Shot-to-shot reproducibility of symmetry conditions is also an important factor in our overall observational sensitivity. A series of implosion shots has been aimed specifically at this issue.

Clusters of shots were taken in sequence with special attention given to quality control of the laser and target fabrication: a) beams were carefully realigned hefore each shot using a recently updated alignment procedure (resulting in less than 30 micron beam pointing error), b) beam balance was carefully adjusted using "precision Nova" techniques (giving < $15 \%$ random power imbalance in the peak of the laser pulse), and c) the targets were carefully selected to minimize target-to-target variations (having less than 1 micron variation in shell thickness). One shot sequence was taken with round compressed core images and one with images (intentionally) distorted in the "pancake" mode. Data for these shots is shown are Figure 11. The Precision Nova techniques that were employed in these experiments will be naturally incorporated into the design of the NIF. These data demonstrate that in addition to sensitive tuning, highly reproducible round and modestly distorted images can be obtained. All data in Figure 11 were taken with shaped laser pulse PS22 and unlined hohlraums. 


\section{CONCLUSIONS}

In the current work the principal conclusions are:

Methods have been demonstrated that are adequate to measure variations in llux asymmetry less than $2 \%$ (integrated over the drive). This is close to the level needed in ignition target designs.

These methods have been applied to symmetry measurement and control experiments and the results have been successfully modeled.

Promising time-resolved techniques have been developed and in some cases have been modeled successfully.

\section{REFERENCES}

1. Hauer, A., ct al. Bull.Am.Phys.Soc. 38, 1884 (1993).

2. Ress, D., et al., Bull.Am.Phys.Soc. 38, 1885 (1993).

3. R. Kauffmann,. F., Ze (1989) private communication and Baker, D., et al., Bull.Am.Phys.Soc. 38, 2083 (1993).

4. Suter, L., Turner, R., (1985) private

communication and Delamater, N., ct al., Bull.Am.Phys.Soc. 38, 1885 (1993).

5. Hammel, B., Bull.Am.Phys.Soc. 38, 2011 (1993).

6. Harris, D., et al. Bull.Am.Phys.Soc. 38 , 1885 (1993).

7. Zimmerman, G., \& Kruer, W., Comments Plasma Physics Controlled Fusion 2, 51 (1975).

\section{ACKNOWLEDGMENTS}

The authors would like to express our appreciation for the essential support of the Nova laser and target operations staffs. We would also like to acknowledge the invaluable role of the Los Alamos technical staff with the crucial assistance of the LLNL technical staff. Jim Murray, Tim Weiland, Bob Ehrlich and John Caird were especially helpful in characterizing and controlling the laser conditions.
We also acknowledge the invaluable and dedicated role of the Los Alamos target fabrication staff and the essential assistance of the L.LNL target fabrication staff in constructing the complicated targets used in this work. Target fabrication quality control from both institutions was critical to the success of these experiments. 
FIGURE CAPTIONS

1. Illumination geometry on Nova. Five beams are incident from each side. (a) view orthogonal to the hohlraum axis; in polar symmetry tuning, the 2 "beam rings" are moved with respect the capsule (variation of "d" in the sketch); (b) view along the hohlraum axis illustrating blowoff from the laser spots nearest the entrance hole.

2. Diagnostic configuration for symmetry measurements using $x$-ray imaging. Two views of an imploded core along orthogonal axes are shown. These images are time gated snapshots $(\sim 80$ ps duration) taken at the peak of emission form the core. The capsi le typically consists of a plastic shell with inner diameter $\sim 440$ microns and about 55 microns thick. The capsule is filled about $50 \mathrm{~atm}$. DD gas and $0.1 \mathrm{~atm}$. Ar.
3. Illustration of flux asymmetry estimation from comparison of modeling to image data. The point on the graph represents the actual data from the image on the left.

4. Illustration of an image decomposition. The image contour is fitted to a Legendre polynomial. 
6. Comparison of modeling to capsule image distortion. (a) Experimental data shown modeling agrees with sensitive symmetry tuning. (b) laser pulse shape designated PS22.

5. Qualitative variations of compressed-core symmetry signatures with beam pointing. 
8. Application of the "symmetry capsule" sampling technique to the evolution for a pure gold hohlraum driven by laser pulse shape 22 . (a) experiment-calculation for later-time implosions. b) experiment-calculation for early-time implosion

7. "Symmetry-capsule" methods for sampling the time varying symmetry in hohlraums. The temporal plots represent a generic variation of the pole to equator drive. The arrows indicate the approximate interval over which the various capsule implosions (coming from different wall thickness capsules) would sample. 
9. The reemission method for symmetry measurement uses a surrogate sphere placed in the hohlraum as shown in (a). In this test of the concept the west cone (cluster of 5 beams) intentional had twice the energy of the east cone. Image data from this shot, given in (b), illustrates early time $x$-ray radiation imbalance. These data were taken with a gated $x$-ray framing camera, and the frame shown was taken about $200 \mathrm{PS}$ into a $1 \mathrm{~ns}$ laser drive.

10. Evolution of hohlraum environment with an intentionally imposed (east - west) laser beam imbalance of $2: 1$. The line-outs for each frame are taken horizontally through the center of the reemission ball. The top frame is taken about 200 ps. after the rise of a $1 \mathrm{~ns}$ laser drive pulse; the middle is about $400 \mathrm{ps}$. after the rise and the lower about $800 \mathrm{ps}$. 

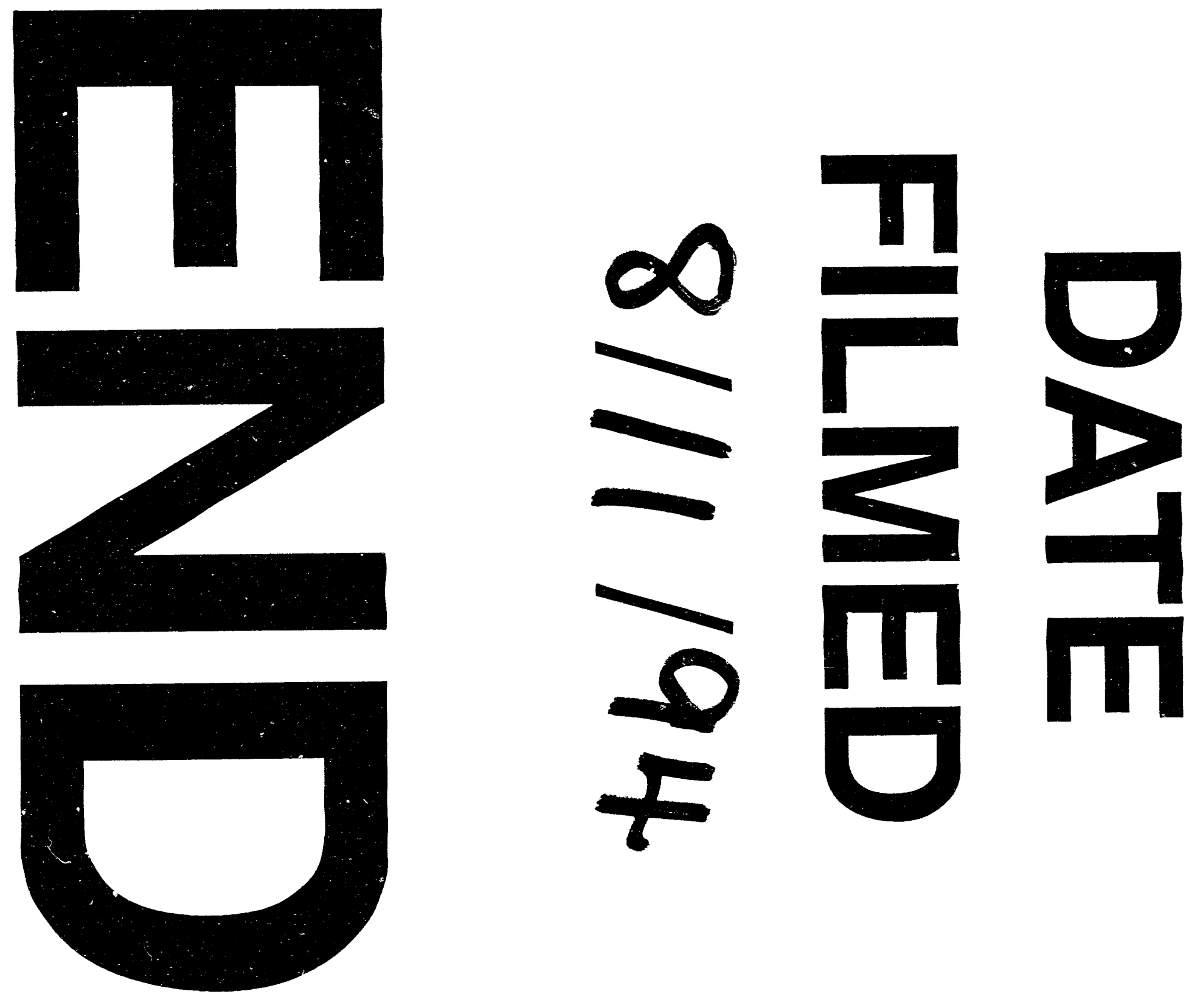